\title{
High-Resolution Magnetic Resonance Imaging Using Compressed Sensing for Intracranial and Extracranial Arteries: Comparison with Conventional Parallel Imaging
}

\author{
Chong Hyun Suh, MD, Seung Chai Jung, MD, PhD, Ho Beom Lee, RT, Se Jin Cho, MD \\ All authors: Department of Radiology and Research Institute of Radiology, University of Ulsan College of Medicine, Asan Medical Center, \\ Seoul, Korea
}

Objective: To compare conventional sensitivity encoding (SENSE) to compressed sensing plus SENSE (CS) for highresolution magnetic resonance imaging (HR-MRI) of intracranial and extracranial arteries.

Materials and Methods: HR-MRI was performed in 14 healthy volunteers. Three-dimensional T1-weighted imaging (T1WI) and proton density-weighted imaging (PD) were acquired using CS or SENSE under the same total acceleration factors $\left(\mathrm{AF}_{\mathrm{t}}\right)$ 5.5, 6.8, and 9.7 for T1WI and 3.2, 4.0, and 5.8 for PD-to achieve reduced scanning times in comparison with the original imaging sequence (SENSE T1WI, AF $\mathrm{F}_{\mathrm{t}} 3.5 ; \mathrm{SENSE} \mathrm{PD,} \mathrm{AF}_{\mathrm{t}} 2.0$ ) using the 3-tesla system. Two neuroradiologists measured signalto-noise ratio (SNR) and contrast-to-noise ratio (CNR), and used visual scoring systems to assess image quality. Acceptable imaging was defined as a visual score $\geq 2$. Repeated measures analysis of variance and Cochran's $Q$ test were performed.

Results: CS yielded better image quality and vessel delineation than SENSE in T1WI with $\mathrm{AF}_{\mathrm{t}}$ of 5.5, 6.8, and 9.7, and in PD with $\mathrm{AF}_{\mathrm{t}}$ of $5.8(p<0.05)$. CS T1WI with $\mathrm{AF}_{\mathrm{t}}$ of 5.5 and CS PD with $\mathrm{AF}_{\mathrm{t}}$ of 3.2 and 4.0 did not differ significantly from original imaging $(p>0.05)$. SNR and CNR in CS were higher than they were in SENSE, but lower than they were in the original images $(p<0.05)$. CS yielded higher proportions of acceptable imaging than SENSE (CS T1WI with $\mathrm{AF}_{\mathrm{t}}$ of 6.8 and PD with $\mathrm{AF}_{\mathrm{t}}$ of 5.8; $p<0.0167)$.

Conclusion: CS is superior to SENSE, and may be a reliable acceleration method for vessel HR-MRI using AF $F_{t}$ of 5.5 for T1WI, and 3.2 and 4.0 for PD.

Keywords: Intracranial artery disease; Image quality; Undersampling; Reconstruction method; Signal-to-noise ratio;

Contrast-to-noise ratio; Compressed sensing

\section{INTRODUCTION}

With its high spatial resolution and excellent soft-tissue

Received July 5, 2018; accepted after revision September 17, 2018.

This study was supported by a grant from the Korea Healthcare Technology R\&D Project, Ministry for Health, Welfare \& Family Affairs, Republic of Korea (HI12C1847).

Corresponding author: Seung Chai Jung, MD, PhD, Department of Radiology and Research Institute of Radiology, University of Ulsan College of Medicine, Asan Medical Center, 88 Olympic-ro 43-gil, Songpa-gu, Seoul 05505, Korea.

- Tel: (822) 3010-3949 - Fax: (822) 476-4719

- E-mail: dynamics79@gmail.com

This is an Open Access article distributed under the terms of the Creative Commons Attribution Non-Commercial License (https://creativecommons.org/licenses/by-nc/4.0) which permits unrestricted non-commercial use, distribution, and reproduction in any medium, provided the original work is properly cited. contrast, high-resolution magnetic resonance imaging (HRMRI) for the visualization of vessel walls and contours is of substantial interest (1). Intracranial vessel wall MRI is a useful adjunct to digital subtraction angiography for differentiating intracranial artery disease and identifying symptomatic non-stenotic disease (1-4). However, the long imaging acquisition time of HR-MRI of the vessel wall is an important issue in daily clinical practice. A long acquisition time can lead to degradation of image quality due to the higher probability of motion artifacts, especially in elderly patients or those who have recently had a stroke. Conventional parallel imaging techniques such as sensitivity encoding (SENSE) or generalized autocalibrating partial parallel acquisition have been developed $(5,6)$; however, they can adversely affect image quality and the visualization of target diseases. Although a two- 
dimensional spatially selective radiofrequency excitation pulse can achieve an acceptable reduction in scan time, this may be achieved at the cost of limited scan coverage (2). Therefore, a novel sequence for vessel HR-MRI is required, to reduce image acquisition time while maintaining image quality and vessel delineation.

Lustig et al. (7) developed compressed sensing for rapid MRI, and demonstrated accelerated acquisition times and improved spatial resolution in multi-slice fast spin echo brain imaging and three-dimensional (3D) contrastenhanced angiography. Compressed sensing enables accelerated MRI by using wavelet transformations and nonlinear iterative reconstruction from variable-density random undersampling of $\mathrm{k}$-space data (7). Conventional parallel imaging techniques also reduce scanning time by undersampling, but the undersampling is done uniformly and images are reconstructed using surface coil information. Different undersampling and reconstruction methods may have different influences on image quality and spatial resolution. Previous studies suggest that compressed sensing can be successfully applied to vessel MRI for intracranial and extracranial arteries, resulting in reduced image acquisition time while maintaining image quality and vessel wall delineation $(8,9)$. To our knowledge however, to date no study investigating the application of compressed sensing to intracranial vessel HR-MRI and evaluating compressed sensing in comparison with conventional parallel imaging (SENSE) has been reported.

The aim of the current study was to compare SENSE with compressed sensing plus SENSE (CS) for HR-MRI of intracranial and extracranial arteries, including the evaluation of a number of different acceleration factors.

\section{MATERIALS AND METHODS}

The relevant Institutional Review Board approved this study, and informed consent was obtained from all subjects.

\section{Study Population}

Fourteen healthy volunteers underwent HR-MRI for delineation of intracranial and extracranial arteries between September 2017 and November 2017. Of these 14 patients, 7 were men (mean age, 58.6 years; age range, 40-67 years) and 7 were women (mean age, 55.3 years; age range, 33-65 years), and the overall mean age was 56.9 years (age range, 33-67 years).

\section{Image Acquisition}

All MRI examinations were performed using a 3T system (Ingenia CX; Philips Medical Systems, Best, the Netherlands) with a 32-channel head coil, and 3D T1-weighted imaging (T1WI) and proton density-weighted imaging (PD) were acquired. CS is a newly developed technique combining compressed sensing and SENSE. For comparison purposes, T1WI with CS and SENSE (total acceleration factors $\left[\mathrm{AF}_{\mathrm{t}}\right]$ 5.5, 6.8, and 9.7), and PD with CS and SENSE $\left(\mathrm{AF}_{\mathrm{t}} 3.2,4.0\right.$, and 5.8) were also acquired to achieve reduced scanning times in comparison with the original imaging sequence (SENSE T1WI with $A F_{t} 3.5$ and SENSE PD with $\mathrm{AF}_{\mathrm{t}}$ 2.0). For the T1WI, compressed sensing factors of 1.6, 2.0, and 2.9 resulted in the CS T1WI having respective $\mathrm{AF}_{\mathrm{t}}$ values of 5.5, 6.8 , and 9.7. For the PD, compressed sensing factors of $1.6,2.0$, and 2.9 resulted in the CS PD having respective $A F_{t}$ values of $3.2,4.0$, and 5.8. The imaging parameters for the original T1WI were echo time $15 \mathrm{~ms}$, repetition time $900 \mathrm{~ms}$, flip angle $90^{\circ}$, matrix $320 \times 320 \times 333$, field of view $192 \times 192 \times 200 \mathrm{~mm}$, voxel size $0.6 \times 0.6 \times 0.6 \mathrm{~mm}$, acceleration factor 3.5, and acquisition time 9 minutes 18 seconds. The imaging parameters for the original PD were echo time $35 \mathrm{~ms}$, repetition time $2000 \mathrm{~ms}$, flip angle $90^{\circ}$, matrix $300 \times 300 \times 75$, field of view $120 \times 120 \times 30 \mathrm{~mm}$, voxel size $0.4 \times 0.4 \times 0.4 \mathrm{~mm}$, acceleration factor 2 , and acquisition time 12 minutes 36 seconds.

The total acquisition times for the T1WI were 9 minutes 18 seconds for the original sequence, 5 minutes 46 seconds (62\% of the original scanning time) for CS and SENSE with $\mathrm{AF}_{\mathrm{t}} 5.5,4$ minutes 39 seconds ( $50 \%$ of the original scanning time) for CS and SENSE with $\mathrm{AF}_{\mathrm{t}} 6.8$, and 3 minutes 15 seconds (35\% of the original scanning time) for CS and SENSE with $\mathrm{AF}_{\mathrm{t}}$ 9.7. The total acquisition times for the PD were 12 minutes 36 seconds for the original sequence, 7 minutes 52 seconds ( $62 \%$ of the original scanning time) for CS and SENSE with $\mathrm{AF}_{\mathrm{t}} 3.2,6$ minutes 18 seconds ( $50 \%$ of the original scanning time) for CS and SENSE with $\mathrm{AF}_{\mathrm{t}}$ 4.0, and 4 minutes 22 seconds (35\% of the original scanning time) for CS and SENSE with $\mathrm{AF}_{\mathrm{t}}$ 5.8.

\section{Image Analysis}

Two neuroradiologists who were blinded to the sequence information, one with 1 year of experience and the other with 7 years of experience independently assessed the images both qualitatively and quantitatively. In qualitative analyses, each T1WI acquisition was rated using a 4-point visual scoring system for image quality and vessel wall 
delineation (10). Overall image quality and artifact were graded as follows: 0 , poor image quality with large artifact; 1 , moderate image quality with moderate artifact; 2 , good image quality with slight artifact; 3 , excellent image quality without artifact (Fig. 1). Vessel wall delineation was graded as follows: 0 , less than $50 \%$ of the vessel wall is visible; 1 , more than $50 \%$ of the vessel wall is visible; 2 , the vessel wall is delineated with adequate signal and contrast to the lumen and cerebrospinal fluid (CSF); 3 , the vessel wall is delineated with excellent signal and sharp contrast to the lumen and CSF (10). Each PD acquisition was rated for image quality using the same scoring system as for the T1WI, and vessel delineation of the outer contour and branching arteries were graded as follows: 0 , less than $50 \%$ of the vessel is visible; 1 , more than $50 \%$ of the vessel is visible; 2, the vessel is delineated with adequate signal and contrast to the lumen and CSF; 3 , the vessel is delineated with excellent signal and sharp contrast to the lumen and CSF (Fig. 2). Acceptable images were defined as those with scores $\geq 2$ for both image quality and vessel delineation.

In quantitative analyses, signal-to-noise ratio (SNR) and contrast-to-noise ratio (CNR) measurements were obtained for the middle cerebral artery, basilar artery, terminal internal carotid artery, and carotid bulb. Digital Imaging and Communications in Medicine data derived from the magnetic resonance images were loaded into the "AsanJ" software developed in-house, which is based on a plug-in package for ImageJ (Bethesda, MD, USA; http:// rsbweb.nih.gov/ij/) for lesion segmentation. The regions of interest (ROIs) used in the analysis were drawn by two

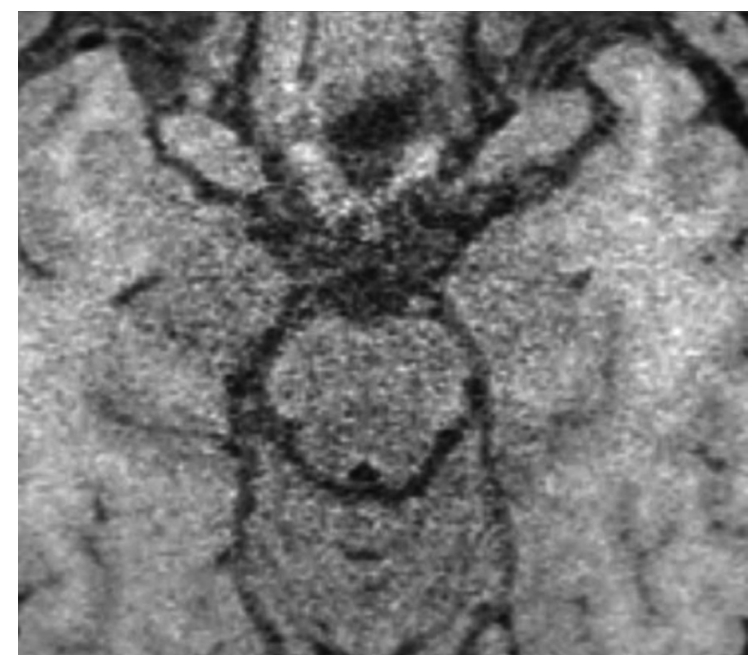

A

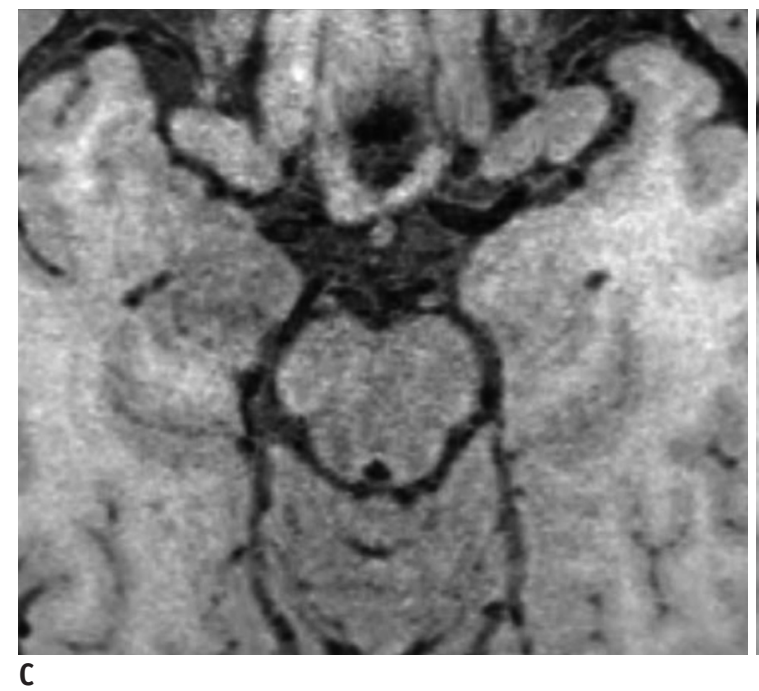

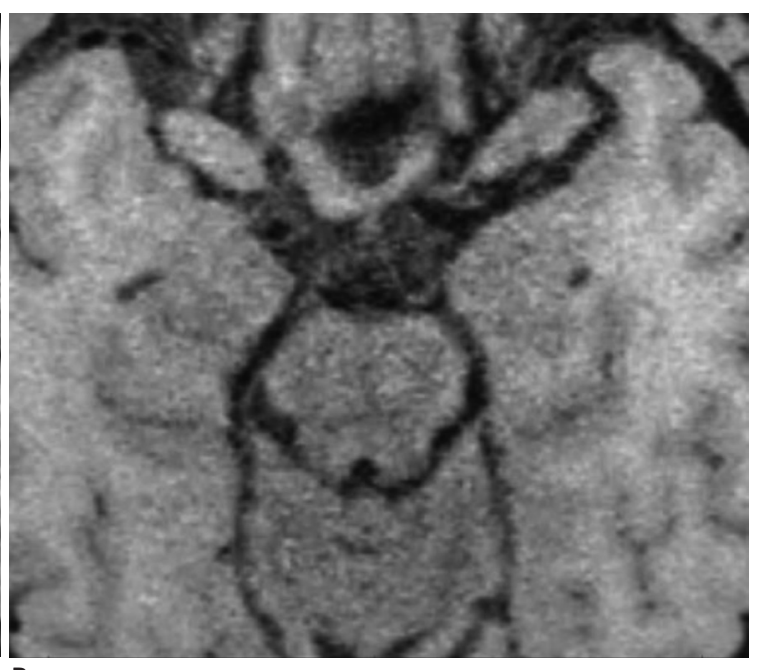

B

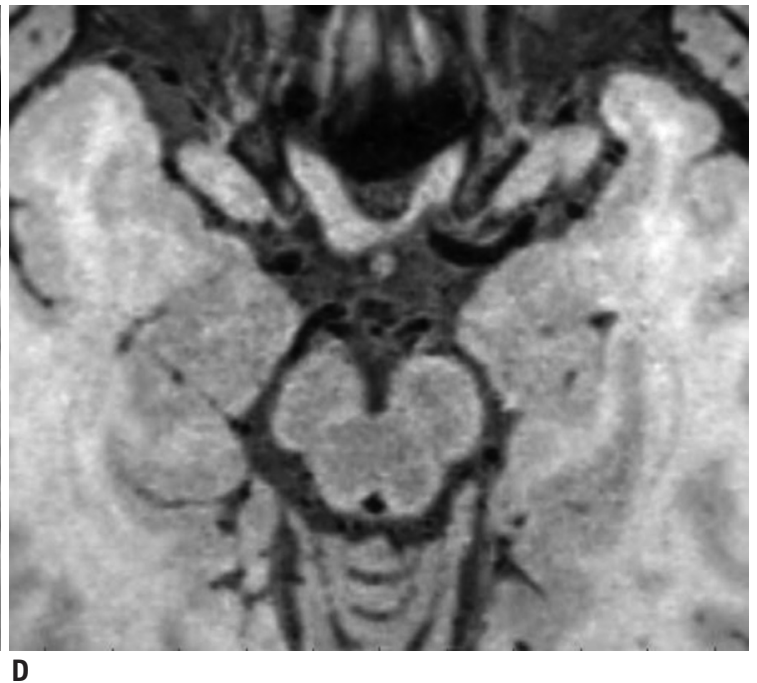

Fig. 1. Overall image quality and artifact were graded as follows.

(A) Grade 0, poor image quality with large artifact, (B) grade 1, moderate image quality with moderate artifact, (C) grade 2, good image quality with slight artifact, and (D) grade 3, excellent image quality without artifact. 

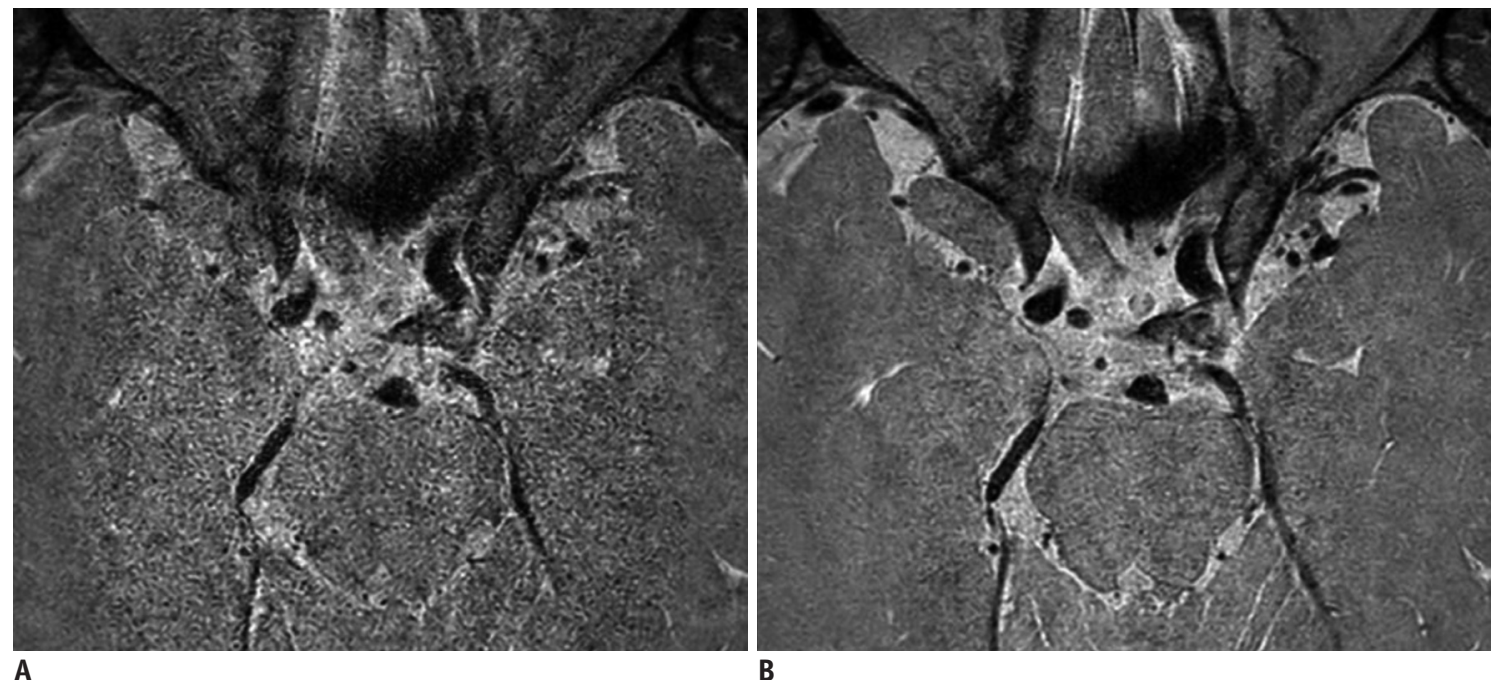

A

B
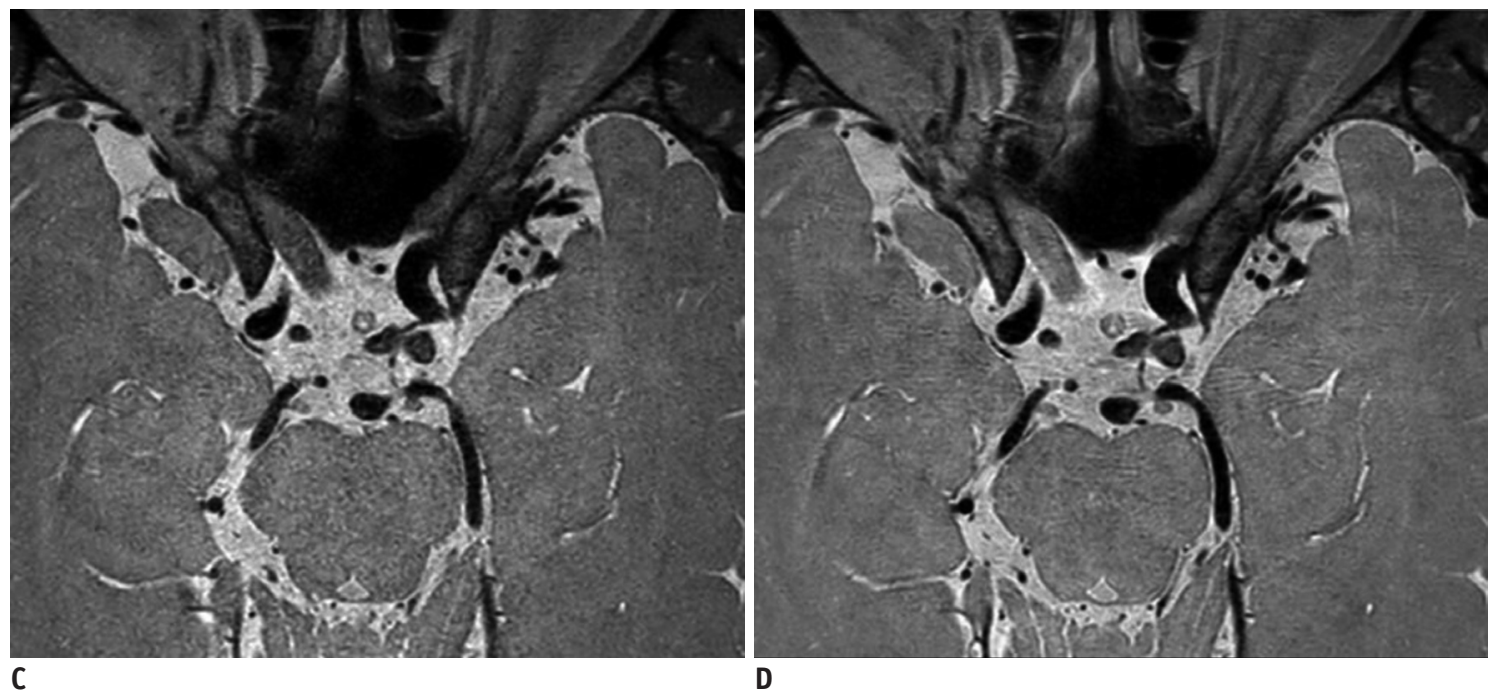

Fig. 2. Vessel delineations of outer contour and branching arteries were graded as follows.

(A) Grade 0 , less than $50 \%$ of vessel is visible, (B) grade 1, more than $50 \%$ of vessel is visible, (C) grade 2, vessel is delineated with adequate signal and contrast to lumen and CSF, and (D) grade 3, vessel is delineated with excellent signal and sharp contrast to lumen and CSF. CSF $=$ cerebrospinal fluid

neuroradiologists. An amplitude segmentation technique based on histogram features and the following formula was used to automatically place the ROIs $(11,12)$ :

$$
r_{i, j}=\left\{\begin{array}{lc}
1, & T_{\min }<p_{i, j} \leq T_{\max } \\
0, & \text { otherwise }
\end{array}\right.
$$

where $r_{i, j}$ is the resulting pixel at coordinate $(i, j), p_{i, j}$ is the corresponding pixel in the input image, and $T$ is the threshold value. When the user places an initial region contour around a vessel wall and double-clicks the mouse, the software automatically calculates the threshold $\left(T_{\text {min, }}\right.$, $\left.T_{\max }\right)$, and delineates the vessel wall to quantify its SNR.

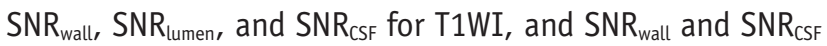
for PD were calculated as follows: SNR $=0.695 \times$ (signal intensity) / (noise), with noise measured as the standard deviation (SD) of the white matter signal (ROI area $>200$ $\mathrm{mm}^{2}$ ). Due to the inhomogeneous noise distribution as a result of parallel imaging, we could not directly measure the noise simply in the air. Instead, we used the SD of the white matter $(13,14)$. CNR wall-lumen $_{\text {and }} \mathrm{CNR}_{\text {wall-CSF }}$ for T1WI, and $\mathrm{CNR}_{\text {cSF-wall }}$ for PD were calculated via the following formula (10):

$$
\mathrm{CNR}_{\mathrm{A}-\mathrm{B}}=\mathrm{SNR}_{\mathrm{A}}-\mathrm{SNR}_{\mathrm{B}}
$$

\section{Statistical Analysis}

We combined the data-sets from both readers and performed all the analyses. For comparisons between 
original, CS, and SENSE images, the Kolmogorov-Smirnov test was used to determine whether the continuous variables were normally distributed. Based on the results of the Kolmogorov-Smirnov test, repeated measures analysis of variance or Friedman tests were used to compare the visual scoring systems, SNR, and CNR of the original, CS, and SENSE images for both intracranial and extracranial arteries. Cochran's $Q$ test was used to compare the proportions of acceptable images of each sequence. Posthoc tests were performed using Bonferroni correction. We also compared acceptability, SNR, and CNR on T1WI for intracranial and extracranial arteries. Interobserver agreements for the original, CS, and SENSE images were assessed using intraclass correlation coefficients (ICCS) and weighted kappa. Two-way random models and consistency assumptions were used for ICC (15). The strength of interobserver agreement was categorized according to the ICC and weighted kappa values as follows: $<0.20$, poor; $0.21-$ 0.40, fair; $0.41-0.60$, moderate; $0.61-0.80$, good; $0.81-1.00$, excellent (16). All statistical analyses were performed using SPSS software (version 20; IBM Corp., Armonk, NY, USA).

\section{RESULTS}

\section{Qualitative Analysis}

For T1WI, CS provided significantly better image quality and vessel wall delineation than SENSE across all $\mathrm{AF}_{\mathrm{t}}$ values $(p<0.05)$. There was no significant difference between the CS sequence with $\mathrm{AF}_{\mathrm{t}} 5.5$ and the original sequence ( $p>$ 0.05 ) (Table 1). CS with $A F_{t} 6.8$ yielded higher proportions of acceptable imaging than SENSE with $\mathrm{AF}_{\mathrm{t}} 6.8(p<$ 0.0167). CS with $A F_{t} 5.5$ and 6.8 and SENSE with $A F_{t} 5.5$ yielded proportions of acceptable imaging that were similar to those of the original sequence $(p>0.05)$ (Table 2). Representative T1WI images are shown in Figure 3.

In PD, CS was significantly superior to SENSE with regard to image quality and vessel delineation for outer contour and branching arteries at $\mathrm{AF}_{\mathrm{t}} 5.8(p<0.05)$. There were no statistically significant differences between the original image, $\mathrm{CS}_{\text {with }} \mathrm{AF}_{\mathrm{t}} 3.2$ and 4.0, and SENSE with $\mathrm{AF}_{\mathrm{t}} 3.2$ and $4.0(p>0.05)$ (Table 1). At an $\mathrm{AF}_{\mathrm{t}}$ of 5.8, CS yielded higher proportions of acceptable imaging than SENSE $(p<0.0167)$. CS with $\mathrm{AF}_{\mathrm{t}} 3.2,4.0$, and 5.8, and SENSE with $\mathrm{AF}_{\mathrm{t}} 3.2$ and 4.0 yielded proportions of acceptable imaging that were similar

Table 1. Comparisons of Visual Scoring Systems on HR-MRI T1WI and PD between Original, CS, and SENSE Imaging

\begin{tabular}{|c|c|c|c|c|c|}
\hline & Acceleration Factor & Original $(95 \% \mathrm{CI})$ & CS $(95 \%$ CI $)$ & SENSE $(95 \% \mathrm{CI})$ & $P$ \\
\hline \multicolumn{6}{|l|}{ T1WI } \\
\hline \multirow[t]{4}{*}{ Image quality } & Original & $2.75(2.55-2.95)$ & & & \\
\hline & 5.5 & & $2.75(2.58-2.92)$ & 2.10 (1.99-2.23) & $<0.001^{\dagger \ddagger}$ \\
\hline & 6.8 & & $2.18(1.99-2.36)$ & $1.71(1.51-1.92)$ & $<0.001^{* \dagger \ddagger}$ \\
\hline & 9.7 & & $1.32(1.14-1.51)$ & $0.46(0.27-0.66)$ & $<0.001^{* \dagger \ddagger}$ \\
\hline \multirow[t]{4}{*}{ Vessel wall delineation } & Original & $2.86(2.72-3.00)$ & & & \\
\hline & 5.5 & & $2.82(2.67-2.97)$ & $2.21(2.05-2.38)$ & $<0.001^{\dagger \ddagger}$ \\
\hline & 6.8 & & $2.25(2.08-2.42)$ & $1.96(1.77-2.16)$ & $<0.001^{* \dagger \ddagger}$ \\
\hline & 9.7 & & $1.07(0.79-1.35)$ & $0.39(0.20-0.59)$ & $<0.001^{* \dagger \ddagger}$ \\
\hline \multicolumn{6}{|l|}{ PD } \\
\hline \multirow[t]{4}{*}{ Image quality } & Original & $2.96(2.89-3.04)$ & & & \\
\hline & 3.2 & & $2.96(2.89-3.04)$ & $2.82(2.64-3.01)$ & 0.182 \\
\hline & 4.0 & & $2.86(2.65-3.06)$ & $2.86(2.72-3.00)$ & 0.432 \\
\hline & 5.8 & & $2.54(2.31-2.76)$ & $1.82(1.56-2.08)$ & $<0.001^{* \dagger \ddagger}$ \\
\hline \multirow[t]{4}{*}{$\begin{array}{l}\text { Vessel outer contour/ } \\
\text { branching arteries } \\
\text { depiction }\end{array}$} & Original & $2.93(2.83-3.03)$ & & & \\
\hline & 3.2 & & $2.93(2.83-3.03)$ & $2.71(2.48-2.95)$ & 0.089 \\
\hline & 4.0 & & $2.79(2.57-3.01)$ & $2.61(2.39-2.83)$ & 0.074 \\
\hline & 5.8 & & $2.04(1.79-2.28)$ & $1.21(0.99-1.43)$ & $<0.001^{* \dagger \ddagger}$ \\
\hline
\end{tabular}

When statistically significant differences were demonstrated by post-hoc tests using Bonferroni correction (Bonferroni corrected $p$ value $<0.05)$, symbols $\left({ }^{*},{ }^{\dagger},{ }^{\ddagger}\right)$ signify $p$ values between original and CS $\left({ }^{*}\right)$, original and SENSE $\left({ }^{\dagger}\right)$, and CS and SENSE $\left({ }^{\ddagger}\right)$. CI $=$ confidence interval, CS = compressed sensing plus SENSE, HR-MRI = high-resolution magnetic resonance imaging, PD = proton density-weighted imaging, SENSE = sensitivity encoding, T1WI $=$ T1-weighted imaging 
Table 2. Comparisons of Acceptable T1WI and PD HR-MRI between Original, CS, and SENSE Imaging

\begin{tabular}{|c|c|c|c|c|c|}
\hline & Acceleration Factor & Original $(95 \% \mathrm{CI})$ & CS $(95 \%$ CI) & SENSE $(95 \% \mathrm{CI})$ & $P$ \\
\hline \multirow[t]{4}{*}{ Intracranial T1WI } & Original & $96.4(81.7-99.9)$ & & & \\
\hline & 5.5 & & $100(87.7-100)$ & $100(87.7-100)$ & 0.368 \\
\hline & 6.8 & & 96.4 (81.7-99.9) & $64.3(44.1-81.4)$ & $<0.001^{\dagger \ddagger}$ \\
\hline & 9.7 & & $21.4(8.3-41.0)$ & $0(0-12.3)$ & $<0.001^{* \dagger}$ \\
\hline \multirow[t]{4}{*}{ Extracranial T1WI } & Original & $100(87.7-100)$ & & & \\
\hline & 5.5 & & $100(87.7-100)$ & $92.9(76.3-99.1)$ & 0.368 \\
\hline & 6.8 & & 92.9 (76.3-99.1) & $71.4(52.8-84.9)$ & $0.002^{\dagger \ddagger}$ \\
\hline & 9.7 & & $7.1(0.9-23.7)$ & $0(0-12.3)$ & $<0.001^{* \dagger}$ \\
\hline \multirow[t]{4}{*}{ PD } & Original & $100(87.7-100)$ & & & \\
\hline & 3.2 & & $100(87.7-100)$ & $92.9(76.6-99.1)$ & 0.135 \\
\hline & 4.0 & & $92.9(76.6-99.1)$ & $96.4(81.7-99.9)$ & 0.368 \\
\hline & 5.8 & & $82.1(63.1-93.9)$ & $28.6(13.2-48.7)$ & $<0.001^{\dagger \ddagger}$ \\
\hline
\end{tabular}

When statistically significant differences were demonstrated by post-hoc tests using Bonferroni correction $(p<0.0167)$, symbols $\left({ }^{*},{ }^{\dagger},{ }^{\ddagger}\right)$ signify $p$ values between original and CS $\left({ }^{*}\right)$, original and SENSE $\left({ }^{\dagger}\right)$, and CS and SENSE $\left({ }^{\ddagger}\right)$.

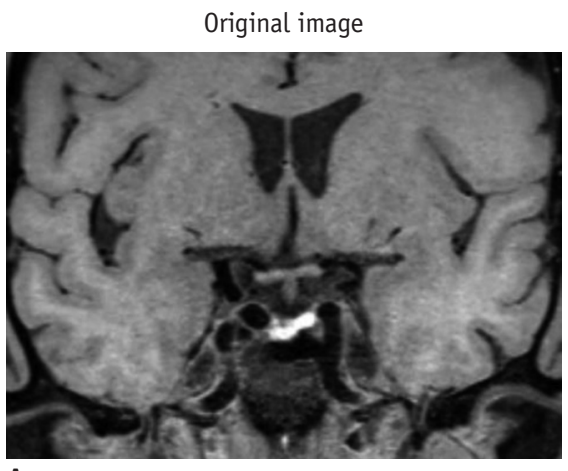

A
CS

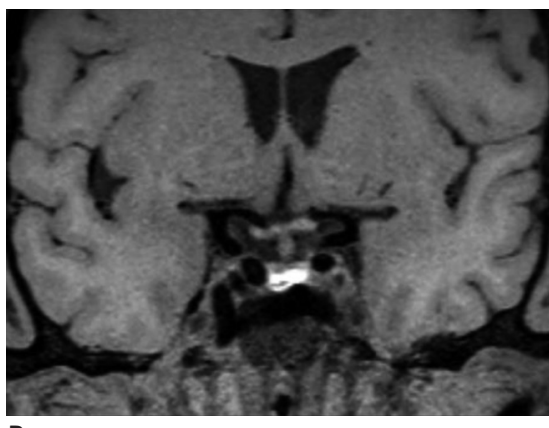

B

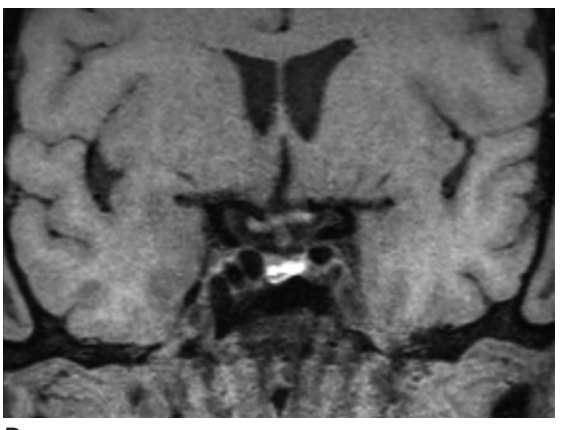

D

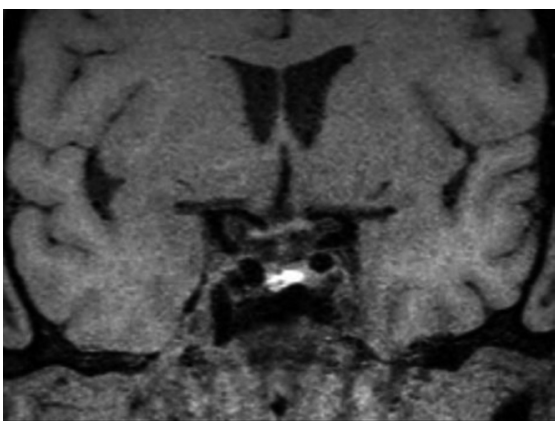

$\mathbf{F}$
SENSE

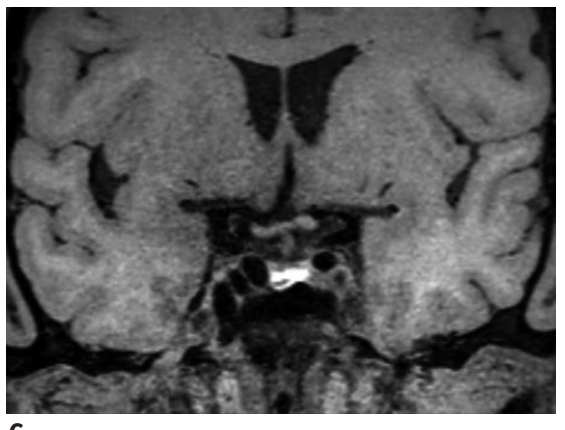

C

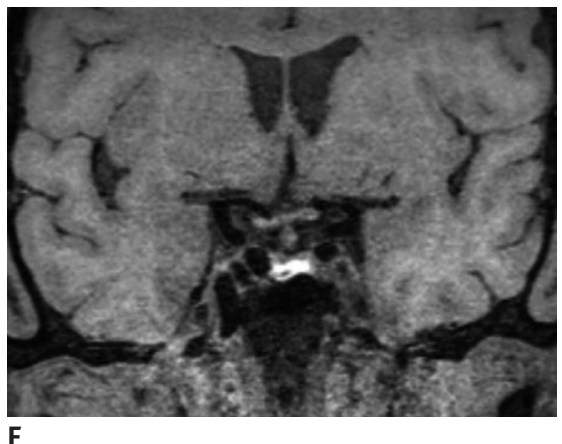

$\stackrel{\rightarrow+}{P}$

io

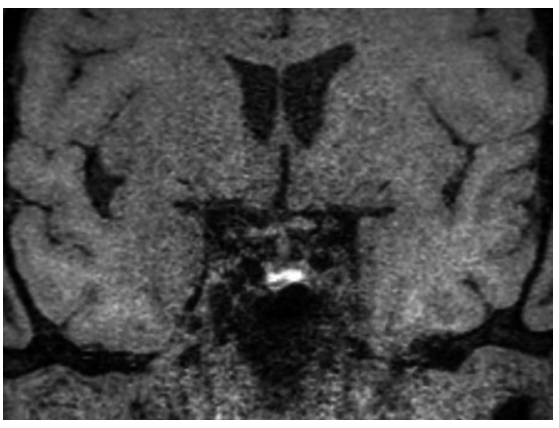

G

Fig. 3. Reconstructed coronal T1-weighted imaging of 59-year-old man. CS exhibits better image quality and vessel wall delineation than SENSE alone at each $\mathrm{AF}_{\mathrm{t}}$. In comparison to original image (A), CS (B, D, F) exhibits better image quality and vessel wall delineation than SENSE alone $(\mathbf{C}, \mathbf{E}, \mathbf{G})$ at each $A F_{t}$. CS (B) and SENSE (C) images with $A_{F_{t}} 5.5$ may be clinically acceptable, whereas SENSE with $\mathrm{AF}_{\mathrm{t}} 9.7(\mathbf{G})$ is uninterpretable. $A F_{t}=$ total acceleration factor, $C S=$ compressed sensing plus SENSE, SENSE = sensitivity encoding 
to those of the original sequences $(p>0.05)$ (Table 2). Separate analyses of acceptability for intracranial and extracranial arteries yielded similar results. Representative PD images are shown in Figure 4.

\section{Quantitative Analysis}

At $\mathrm{AF}_{\mathrm{t}} 5.5,6.8$, and 9.7, $\mathrm{SNR}_{\text {wall }}$ and $\mathrm{CNR}_{\text {wall-lumen }}$ values on T1WI were significantly higher with CS than with SENSE ( $p$
$<0.05)$. SNR $R_{\text {lumen }}$ with $C S$ and $A F_{t} 9.7$, and SNR $R_{\text {CSF }}$ with $C S$ and $A F_{t} 6.8$ and 9.7 were significantly higher than they were with SENSE $(p<0.05)$. On PD, SNR CSF with CS and $\mathrm{AF}_{\mathrm{t}} 5.8$, and $\mathrm{CNR}_{\text {CSF-wall }}$ with $\mathrm{CS}$ and $\mathrm{AF}_{\mathrm{t}} 4.0$ and 5.8 were significantly higher than they were with SENSE $(p<0.05)$ (Table 3$)$. Separate analyses of intracranial and extracranial arteries yielded similar results (Table 4). At AFt 5.5 and 9.7, $\mathrm{SNR}_{\text {wall }}$ values on both intracranial and extracranial T1WI were

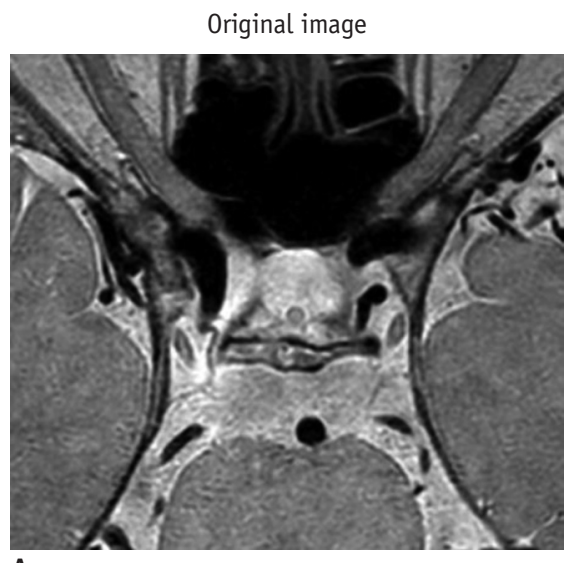

A

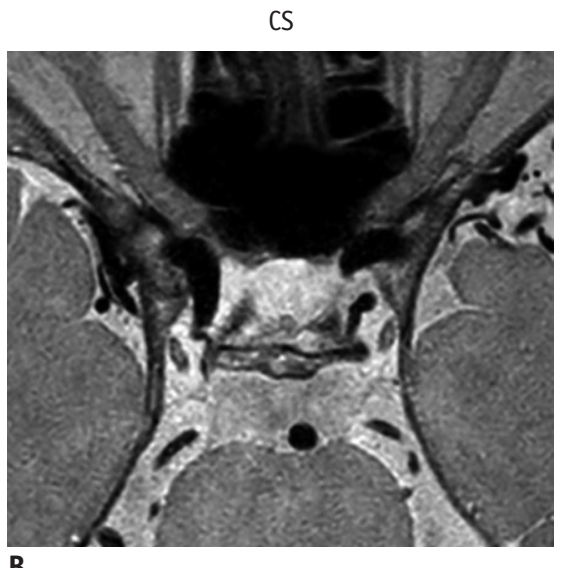

B

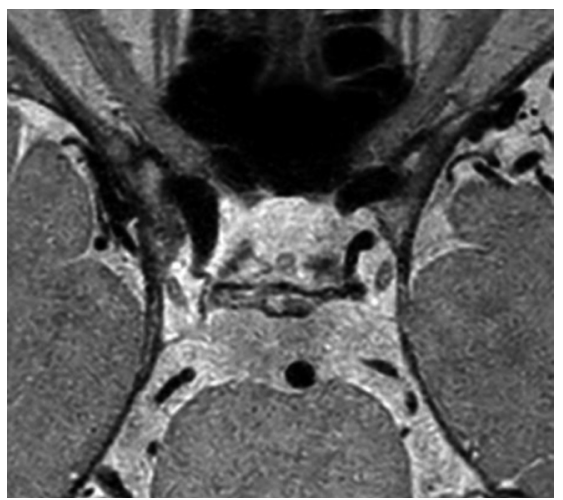

D

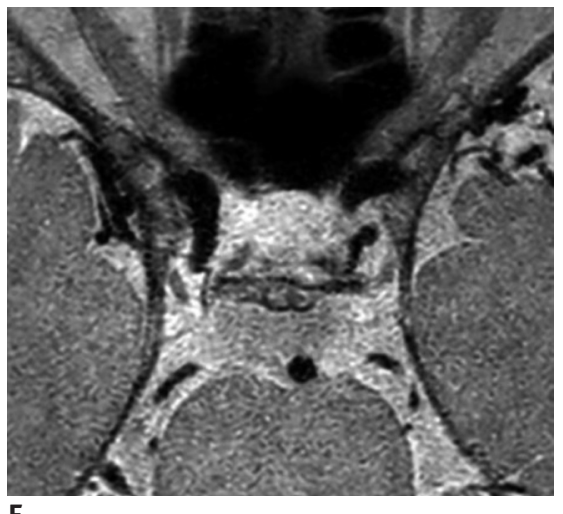

$\mathbf{F}$
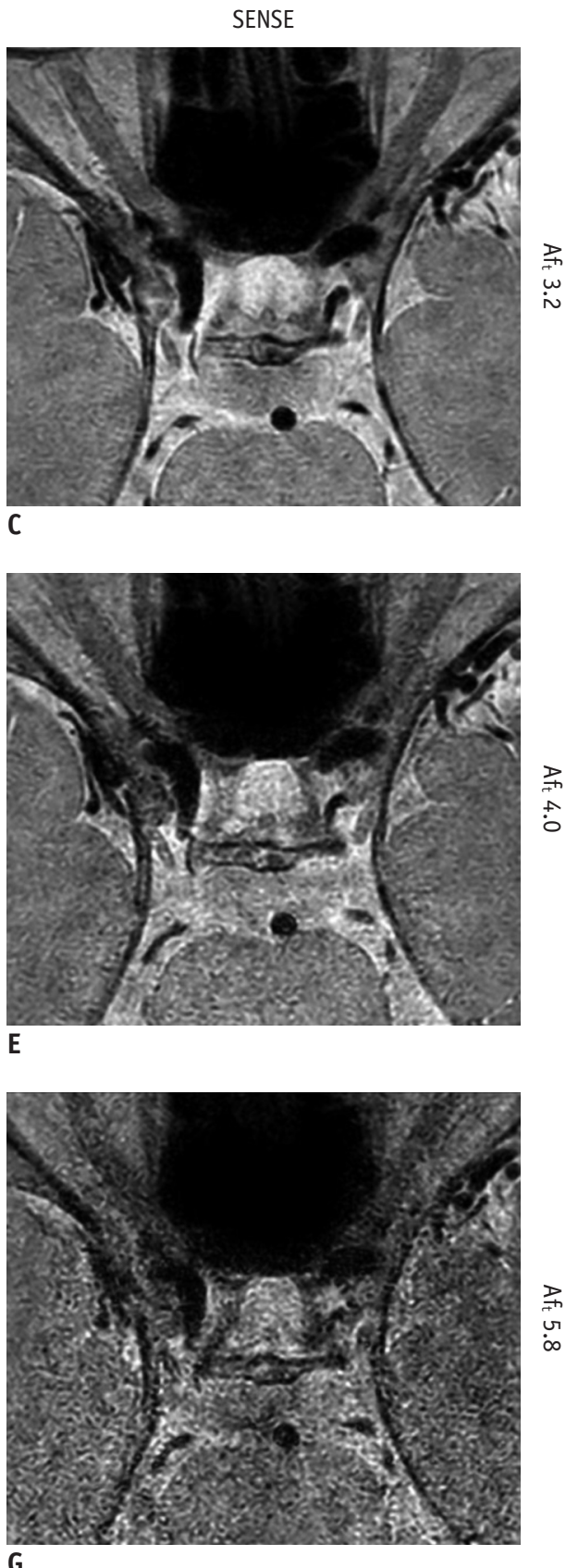

Fig. 4. Axial proton density-weighted imaging of 58-year-old man. CS yielded better image quality and vessel delineation for outer contour and branching arteries than SENSE alone at each $\mathrm{AF}_{\mathrm{t}}$. In comparison to original image $(\mathbf{A}), \mathbf{C S}(\mathbf{B}, \mathbf{D}, \mathbf{F})$ yielded better image quality and vessel delineation for outer contour and branching arteries than SENSE alone $(\mathbf{C}, \mathbf{E}, \mathbf{G})$ at each $\mathrm{AF}_{\mathrm{t}}$. CS (B, D) and SENSE $(\mathbf{C}, \mathbf{E})$ images with $A F_{t} 3.2$ and 4.0 may be clinically acceptable, whereas SENSE with $\mathrm{AF}_{\mathrm{t}} 5.8(\mathbf{G})$ is uninterpretable. 
Table 3. Comparisons of SNR and CNR between Original, CS, and SENSE Imaging for T1WI and PD HR-MRI

\begin{tabular}{|c|c|c|c|c|c|}
\hline & Acceleration Factor & Original $(95 \% \mathrm{CI})$ & CS $(95 \%$ CI $)$ & SENSE $(95 \% \mathrm{CI})$ & $P$ \\
\hline \multicolumn{6}{|l|}{ T1WI } \\
\hline \multirow[t]{4}{*}{$\mathrm{SNR}_{\text {wall }}$} & Original & $12.29(11.63-12.96)$ & & & \\
\hline & 5.5 & & $10.67(10.07-11.28)$ & $10.06(9.52-10.61)$ & $<0.001^{* \dagger \ddagger}$ \\
\hline & 6.8 & & $8.61(8.13-9.08)$ & $8.18(7.75-8.60)$ & $<0.001^{* \dagger \ddagger}$ \\
\hline & 9.7 & & $6.56(6.20-6.92)$ & $5.26(4.99-5.53)$ & $<0.001^{* \dagger \ddagger}$ \\
\hline \multirow[t]{4}{*}{ SNR lumen } & Original & $3.35(3.19-3.52)$ & & & \\
\hline & 5.5 & & $2.94(2.78-3.10)$ & $2.86(2.71-3.01)$ & $<0.001^{* \dagger}$ \\
\hline & 6.8 & & $2.51(2.39-2.63)$ & $2.55(2.44-2.66)$ & $<0.001^{* \dagger}$ \\
\hline & 9.7 & & $2.16(2.07-2.26)$ & $1.96(1.86-2.06)$ & $<0.001^{* \dagger \ddagger}$ \\
\hline \multirow[t]{4}{*}{ SNR } & Original & $7.51(7.03-7.99)$ & & & \\
\hline & 5.5 & & $6.79(6.33-7.25)$ & $6.23(5.63-6.83)$ & $0.002^{\dagger}$ \\
\hline & 6.8 & & $5.77(5.39-6.15)$ & $5.26(4.95-5.57)$ & $<0.001^{* \dagger \ddagger}$ \\
\hline & 9.7 & & $4.38(4.15-4.61)$ & $2.97(2.80-3.13)$ & $<0.001^{* \dagger \ddagger}$ \\
\hline \multirow[t]{4}{*}{$\mathrm{CNR}_{\text {wall-lumen }}$} & Original & $8.94(8.32-9.55)$ & & & \\
\hline & 5.5 & & $7.73(7.20-8.26)$ & $7.20(6.72-7.68)$ & $<0.001^{* \dagger \neq}$ \\
\hline & 6.8 & & $6.10(5.65-6.54)$ & $5.63(5.24-6.01)$ & $<0.001^{* \dagger \ddagger}$ \\
\hline & 9.7 & & $4.40(4.05-4.74)$ & $3.30(3.06-3.54)$ & $<0.001^{* \dagger \ddagger}$ \\
\hline \multirow[t]{4}{*}{$\mathrm{CNR}_{\text {wall-CSF }}$} & Original & $4.78(4.15-5.41)$ & & & \\
\hline & 5.5 & & $3.95(3.38-4.53)$ & $3.76(3.23-4.29)$ & $<0.001^{* \dagger}$ \\
\hline & 6.8 & & $2.85(2.41-3.29)$ & $2.90(2.46-3.34)$ & $<0.001^{* \dagger}$ \\
\hline & 9.7 & & $2.19(1.88-2.51)$ & $2.29(1.99-2.58)$ & $<0.001^{* \dagger}$ \\
\hline \multicolumn{6}{|l|}{ PD } \\
\hline \multirow[t]{4}{*}{$\mathrm{SNR}_{\text {wall }}$} & Original & $3.01(2.83-3.20)$ & & & \\
\hline & 3.2 & & $2.67(2.50-2.83)$ & $2.48(2.30-2.66)$ & $<0.001^{* \dagger}$ \\
\hline & 4.0 & & $2.34(2.15-2.53)$ & $2.36(2.22-2.51)$ & $<0.001^{* \dagger}$ \\
\hline & 5.8 & & $2.12(1.97-2.27)$ & $2.30(2.16-2.45)$ & $<0.001^{* \dagger}$ \\
\hline \multirow[t]{4}{*}{ SNR } & Original & $20.51(19.56-21.47)$ & & & \\
\hline & 3.2 & & $16.54(15.78-17.30)$ & $16.11(15.23-17.09)$ & $<0.001^{* \dagger}$ \\
\hline & 4.0 & & $13.26(12.58-13.94)$ & $12.39(11.60-13.17)$ & $<0.001^{* \dagger}$ \\
\hline & 5.8 & & $11.34(10.81-11.87)$ & $8.93(8.24-9.61)$ & $<0.001^{* \dagger \ddagger}$ \\
\hline \multirow[t]{4}{*}{ CNR $_{\text {cSF-wall }}$} & Original & $17.50(16.50-18.23)$ & & & \\
\hline & 3.2 & & $13.88(13.48-14.27)$ & $13.63(13.12-14.14)$ & $<0.001^{* \dagger}$ \\
\hline & 4.0 & & $10.92(10.55-11.29)$ & $10.03(9.62-10.44)$ & $<0.001^{* \dagger \ddagger}$ \\
\hline & 5.8 & & $9.22(8.96-9.47)$ & $6.62(6.30-6.94)$ & $<0.001^{* \dagger \ddagger}$ \\
\hline
\end{tabular}

When statistically significant differences were demonstrated by post-hoc tests using Bonferroni correction (Bonferroni corrected $p$ value $<0.05)$, symbols $\left({ }^{*},{ }^{\dagger},{ }^{\ddagger}\right)$ signify $p$ values between original and CS $\left({ }^{*}\right)$, original and SENSE $\left({ }^{\dagger}\right)$, and CS and SENSE $\left({ }^{\ddagger}\right)$. CNR $=$ contrast-tonoise ratio, $\mathrm{CSF}=$ cerebrospinal fluid, SNR $=$ signal-to-noise ratio

significantly higher with CS than with SENSE $(p<0.05)$. At $\mathrm{AF}_{\mathrm{t}}$ 5.5, 6.8, and 9.7, $\mathrm{CNR}_{\text {wall-lumen }}$ values on intracranial T1WI were significantly higher with CS than with SENSE $(p<0.05)$. At $A F_{t}$ 9.7, $C^{2} R_{\text {wall-lumen }}$ values on extracranial T1WI were significantly higher with CS than with SENSE $(p<0.05)$.

\section{Interobserver Agreement}

There was good to excellent interobserver agreement between the two observers with regard to the visual scoring system (weighted kappa, 0.784-0.803) and measurements of SNR (ICC, 0.951-0.991) and CNR (ICC, 0.857-0.946).

\section{DISCUSSION}

In the current study, HR-MRI of intracranial and extracranial vessels using CS was superior to that using SENSE, yielding higher image quality, better vessel delineation, SNR, and CNR, and a greater proportion of images deemed acceptable. In comparison with the original sequence, CS may be a reliable method for vessel HR-MRI, with comparable image quality and vessel delineation even though T1WI images were acquired in $62 \%$ of the original image acquisition time, and PD images were acquired in 
Table 4. Comparisons of SNR and CNR between Original, CS, and SENSE Imaging for Intracranial and Extracranial T1WI

\begin{tabular}{|c|c|c|c|c|c|}
\hline & Acceleration Factor & Original (95\% CI) & CS $(95 \%$ CI) & SENSE $(95 \% \mathrm{CI})$ & $P$ \\
\hline \multicolumn{6}{|c|}{ Intracranial T1WI } \\
\hline \multirow[t]{4}{*}{$\mathrm{SNR}_{\text {wall }}$} & Original & $11.58(10.84-12.31)$ & & & \\
\hline & 5.5 & & $9.89(9.23-10.55)$ & $9.28(8.70-9.86)$ & $<0.001^{* \dagger \ddagger}$ \\
\hline & 6.8 & & 7.87 (7.37-8.37) & $7.45(7.02-7.88)$ & $<0.001^{* \dagger}$ \\
\hline & 9.7 & & $6.03(5.65-6.41)$ & $4.81(4.53-5.10)$ & $<0.001^{* \dagger \ddagger}$ \\
\hline \multirow[t]{4}{*}{ SNR } & Original & $3.23(3.05-3.40)$ & & & \\
\hline & 5.5 & & $2.78(2.62-2.94)$ & $2.73(2.57-2.88)$ & $<0.001^{* \dagger}$ \\
\hline & 6.8 & & $2.49(2.35-2.63)$ & $2.48(2.37-2.60)$ & $<0.001^{* \dagger}$ \\
\hline & 9.7 & & $2.13(2.02-2.25)$ & $1.85(1.75-1.94)$ & $<0.001^{* \dagger \ddagger}$ \\
\hline \multirow[t]{4}{*}{$\mathrm{CNR}_{\text {wall-lumen }}$} & Original & $8.35(7.68-9.02)$ & & & \\
\hline & 5.5 & & $7.11(6.52-7.69)$ & $6.55(6.04-7.06)$ & $<0.001^{* \dagger \ddagger}$ \\
\hline & 6.8 & & $5.38(4.92-5.83)$ & $4.97(4.58-5.36)$ & $<0.001^{\star \dagger \ddagger}$ \\
\hline & 9.7 & & $3.90(3.54-4.27)$ & $2.97(2.71-3.23)$ & $<0.001^{* \dagger \ddagger}$ \\
\hline \multirow[t]{4}{*}{$\mathrm{CNR}_{\text {wall-CSF }}$} & Original & $4.07(3.37-4.77)$ & & & \\
\hline & 5.5 & & $3.14(2.50-3.78)$ & $3.00(2.42-3.59)$ & $<0.001^{* \dagger}$ \\
\hline & 6.8 & & $2.12(1.67-2.58)$ & $2.17(1.71-2.64)$ & $<0.001^{* \dagger}$ \\
\hline & 9.7 & & $1.66(1.34-1.99)$ & $1.84(1.53-2.16)$ & $<0.001^{* \dagger}$ \\
\hline \multicolumn{6}{|c|}{ Extracranial T1WI } \\
\hline \multirow[t]{4}{*}{$\mathrm{SNR}_{\text {wall }}$} & Original & $14.44(13.20-15.68)$ & & & \\
\hline & 5.5 & & $13.03(12.04-14.02)$ & $12.42(11.55-13.28)$ & $<0.001^{\dagger}$ \\
\hline & 6.8 & & $10.83(10.10-11.56)$ & $10.35(9.75-10.94)$ & $<0.001^{* \dagger}$ \\
\hline & 9.7 & & $8.14(7.56-8.71)$ & $6.61(6.26-6.96)$ & $<0.001^{* \dagger \ddagger}$ \\
\hline \multirow[t]{4}{*}{$\mathrm{SNR}_{\text {lumen }}$} & Original & $3.73(3.32-4.13)$ & & & \\
\hline & 5.5 & & $3.42(3.03-3.82)$ & $3.25(2.91-3.60)$ & $<0.001^{\dagger}$ \\
\hline & 6.8 & & $2.56(2.32-2.81)$ & $2.76(2.47-3.05)$ & $<0.001^{* \dagger}$ \\
\hline & 9.7 & & $2.27(2.08-2.45)$ & $2.31(2.10-2.51)$ & $<0.001^{* \dagger}$ \\
\hline \multirow[t]{4}{*}{$\mathrm{CNR}_{\text {wall-lumen }}$} & Original & $10.71(9.46-11.96)$ & & & \\
\hline & 5.5 & & $9.60(8.70-10.51)$ & $9.16(8.34-9.98)$ & $<0.001^{\dagger}$ \\
\hline & 6.8 & & $8.27(7.58-8.95)$ & $7.59(6.96-8.21)$ & $<0.001^{* \dagger}$ \\
\hline & 9.7 & & $5.87(5.29-6.45)$ & $4.31(3.91-4.70)$ & $<0.001^{* \dagger \ddagger}$ \\
\hline
\end{tabular}

When statistically significant differences were demonstrated by post-hoc tests using Bonferroni correction (Bonferroni corrected $p$ value $<0.05)$, symbols $\left({ }^{*},{ }^{\dagger},{ }^{\ddagger}\right)$ signify $p$ values between original and CS $\left({ }^{*}\right)$, original and SENSE $\left(^{\dagger}\right)$, and CS and SENSE $\left(^{\ddagger}\right)$.

$50 \%$ of the original image acquisition time. CS also yielded acceptable T1WI images at $A F_{t} 5.5$ and 6.8 , and acceptable $P D$ images at $A F_{t} 3.2,4.0$, and 5.8.

Reducing the imaging acquisition time may be crucial for expanding the clinical use of vessel wall HR-MRI in daily practice. Of the various sparse reconstruction techniques available, it has been proposed that CS has promise for intracranial and extracranial vessel wall MRI because it can markedly reduce image acquisition time while preserving image quality. In CS, undersampling of k-space data is achieved via a variable-density random method, which means denser sampling in the central $k$-space than in the peripheral $k$-space $(17,18)$. The sparsity in the k-space data is compensated for by a sparsifying transformation-such as a wavelet transformation-and iterative reconstruction
(17). Differences in the undersampling of k-space data and the specified reconstruction methods may contribute to improved image quality and reduced scanning time compared with conventional parallel imaging. Nonlinear iterative reconstruction has been used to reconstruct magnetic resonance images from fewer phase encodings, thereby resulting in a marked reduction in image acquisition time (7).

A previous study demonstrated that a black-blood fast spin echo sequence with CS facilitated a $37 \%$ shorter image acquisition time than conventional T1-weighted sampling perfection with application optimized contrasts using different flip angle evolution, while maintaining comparable image quality for intracranial arteries (9). In the present study, T1WI with CS also yielded image quality 
and vessel wall delineation that were comparable to those of original imaging sequences, with an image acquisition time that was $38 \%$ shorter ( $62 \%$ of the original scanning time). In another study, CS-based simultaneous 3D blackblood and gray-blood imaging yielded image quality that was comparable to that of a fully sampled acquisition for carotid artery visualization, and achieved it in a shorter scanning time (7). The authors stated that the "signalto-tissue" and "contrast-to-tissue" ratios did not differ significantly between CS images with $\mathrm{AF}_{\mathrm{t}}$ values of 2-5 and original images. In contrast, in the current study SNR and CNR were significantly lower in CS images than in original images, even though image quality and vessel delineation did not differ significantly between them based on results derived from the visual scoring systems. The differences between the results of the two studies may be due to the parameters chosen. The "signal-to-tissue" and "contrast-totissue" ratios may reflect the visual assessment more than the quantitative measurements used in the current study (i.e., SNR and CNR).

CS yielded image quality and vessel delineation that were comparable to the original sequences in the present study, with a reduction in image acquisition time of $50 \%$ for PD. Vessel HR-MRI generally focused on evaluating arterial walls using T1WI; however, PD imaging with a high SNR also provides useful information on definite vessel contours, detailed vessel anatomy, and small branching arteries $(1,19)$. Therefore, the use of CS PD sequences for vessel evaluation is worthy of further assessment. To our knowledge, to date no previous study has evaluated CS PD sequences for intracranial vessel HR-MRI.

In the present study, CS T1WI with $\mathrm{AF}_{\mathrm{t}} 5.5$ and 6.8 , and SENSE T1WI with $A F_{t} 5.5$ yielded respective acceptability rates of $100 \%, 96.4 \%$, and $100 \%$, while CS PD with $\mathrm{AF}_{\mathrm{t}} 3.2$, 4.0, and 5.8, and SENSE PD with $A F_{t} 3.2$ and 4.0 yielded respective acceptability rates of $100 \%, 92.9 \%, 82.1 \%$, $92.9 \%$, and $96.4 \%$. At these $\mathrm{AF}_{\mathrm{t}}$ values, the proportions of acceptable images did not differ significantly from those of the original imaging. According to results derived via the visual scoring systems, acceptable images could be acquired at higher $\mathrm{AF}_{\mathrm{t}}$ values than would otherwise result in image quality comparable to the original sequences. Thus, in terms of clinical acceptability there may be scope to further shorten the acquisition times for both CS and SENSE sequences.

The present study had several limitations. First, it only included 14 healthy volunteers. Further studies including more patients and various clinical applications are required. Second, even the original T1WI and PD sequences included acceleration factors, which may be a limitation with regard to comparisons between original images and CS and SENSE images. Notably however, in clinical practice vessel wall HR-MRI generally includes an acceleration factor to reduce scanning time, and a previous study also compared CS images with original images that included acceleration factors (9). Furthermore, we wished to focus on comparing clinically-used original imaging sequences with CS and SENSE imaging. Third, due to limits on available acquisition time, we only evaluated three different acceleration factors. Further efforts to optimize scanning parameters for CS HR-MRI of vessels are warranted. Fourth, because we only evaluated CS (i.e., compressed sensing combined with SENSE) compared to SENSE alone, it is unclear how the image quality of CS compares with that of compressed sensing alone at the same $A F_{t}$ factors. Further study is warranted in this respect. Fifth, the study did not generate data for contrast-enhanced T1WI, because the use of gadolinium-based contrast agents is not justified in healthy volunteers.

In conclusion, CS was superior to SENSE with regard to image quality, vessel delineation, SNR, CNR, and acceptability in vessel HR-MRI. CS T1WI with $\mathrm{AF}_{\mathrm{t}}$ of 5.5 and CS PD with $\mathrm{AF}_{\mathrm{t}}$ of 3.2 and 4.0 may be reliable for HR-MRI of vessels.

\section{Conflicts of Interest}

The authors have no financial conflicts of interest.

ORCID

Seung Chai Jung

https://orcid.org/0000-0001-5559-7973

Chong Hyun Suh

https://orcid.org/0000-0002-4737-0530

\section{REFERENCES}

1. Mandell DM, Mossa-Basha M, Qiao Y, Hess CP, Hui F, Matouk C, et al.; Vessel Wall Imaging Study Group of the American Society of Neuroradiology. Intracranial vessel wall MRI: principles and expert consensus recommendations of the American Society of Neuroradiology. AJNR Am J Neuroradiol 2017;38:218-229

2. Chung MS, Jung SC, Kim SO, Kim HS, Choi CG, Kim SJ, et al. Intracranial artery steno-occlusion: diagnosis by using twodimensional spatially selective radiofrequency excitation 
pulse MR imaging. Radiology 2017;284:834-843

3. Lee NJ, Chung MS, Jung SC, Kim HS, Choi CG, Kim SJ, et al. Comparison of high-resolution MR imaging and digital subtraction angiography for the characterization and diagnosis of intracranial artery disease. AJNR Am J Neuroradiol 2016;37:2245-2250

4. Park JE, Jung SC, Lee SH, Jeon JY, Lee JY, Kim HS, et al. Comparison of 3D magnetic resonance imaging and digital subtraction angiography for intracranial artery stenosis. Eur Radiol 2017;27:4737-4746

5. Saam T, Raya JG, Cyran CC, Bochmann K, Meimarakis G, Dietrich 0 , et al. High resolution carotid black-blood 3T MR with parallel imaging and dedicated 4-channel surface coils. $J$ Cardiovasc Magn Reson 2009;11:41

6. Itskovich VV, Mani V, Mizsei G, Aguinaldo JG, Samber DD, Macaluso F, et al. Parallel and nonparallel simultaneous multislice black-blood double inversion recovery techniques for vessel wall imaging. J Magn Reson Imaging 2004;19:459-467

7. Lustig M, Donoho D, Pauly JM. Sparse MRI: the application of compressed sensing for rapid MR imaging. Magn Reson Med 2007;58:1182-1195

8. Li B, Li H, Kong H, Dong L, Zhang J, Fang J. Compressed sensing based simultaneous black- and gray-blood carotid vessel wall MR imaging. Magn Reson Imaging 2017;38:214-223

9. Zhu C, Tian B, Chen L, Eisenmenger L, Raithel E, Forman C, et al. Accelerated whole brain intracranial vessel wall imaging using black blood fast spin echo with compressed sensing (CS-SPACE). MAGMA 2018;31:457-467

10. Fan Z, Yang Q, Deng Z, Li Y, Bi X, Song S, et al. Whole-brain intracranial vessel wall imaging at 3 Tesla using cerebrospinal fluid-attenuated T1-weighted 3D turbo spin echo. Magn Reson
Med 2017;77:1142-1150

11. Ramesh N, Yoo JH, Sethi IK. Thresholding based on histogram approximation. IEE P-Vis Image Sign 1995;142:271-279

12. Raju PDR, Neelima G. Image segmentation by using histogram thresholding. IJCSET 2012;2:776-779

13. Qiao Y, Steinman DA, Qin Q, Etesami M, Schär M, Astor BC, et al. Intracranial arterial wall imaging using three-dimensional high isotropic resolution black blood MRI at 3.0 Tesla. J Magn Reson Imaging 2011;34:22-30

14. Zhang Z, Fan Z, Carroll TJ, Chung Y, Weale P, Jerecic R, et al. Three-dimensional T2-weighted MRI of the human femoral arterial vessel wall at 3.0 Tesla. Invest Radiol 2009;44:619-626

15. Park JE, Han K, Sung YS, Chung MS, Koo HJ, Yoon HM, et al. Selection and reporting of statistical methods to assess reliability of a diagnostic test: conformity to recommended methods in a peer-reviewed journal. Korean $\mathrm{J}$ Radiol 2017;18:888-897

16. Kang HJ, Lee JM, Joo I, Hur BY, Jeon JH, Jang JY, et al. Assessment of malignant potential in intraductal papillary mucinous neoplasms of the pancreas: comparison between multidetector CT and MR imaging with MR cholangiopancreatography. Radiology 2016;279:128-139

17. Yang AC, Kretzler M, Sudarski S, Gulani V, Seiberlich N. Sparse reconstruction techniques in magnetic resonance imaging: methods, applications, and challenges to clinical adoption. Invest Radiol 2016;51:349-364

18. Li G, Hennig J, Raithel E, Büchert M, Paul D, Korvink JG, et al. An L1-norm phase constraint for half-Fourier compressed sensing in 3D MR imaging. MAGMA 2015;28:459-472

19. Choi YJ, Jung SC, Lee DH. Vessel wall imaging of the intracranial and cervical carotid arteries. J Stroke 2015;17:238-255 